\title{
ANALYTICAL SOLUTION OF THE MOTION OF THE PLANETS OVER SEVERAL THOUSANDS OF YEARS
}

\author{
P. BRETAGNON \\ Bureau des Longitudes \\ 77, avenue Denfert-Rochereau, 75014 Paris, France
}

\section{Introduction}

The results of a planetary theory built by an iterative method are given here in order to show the relation with the secular variation theories and the meaning of the mean elements in these latter theories. The general theories have a validity span of several millions years but a weak precision; on the contrary, the secular variation theories reach a great precision over several thousand years. Two applications of the analytical planetary theories are presented : the relation between the barycentric coordinates and the geocentric ones; the determination of the terms of precession and nutation for the rigid Earth.

\section{General theories}

\subsection{FORM OF THE SOLUTION}

The general theories represent the motion of the planets in Fourier series :

$$
\begin{aligned}
a_{i}= & a_{i 0}+\sum_{\Phi^{*}} A_{i, \Phi^{*}} \cos \Phi^{*}+\sum_{\Phi} A_{i, \Phi} \cos \Phi \\
\lambda_{i}= & \lambda_{i 0}+n_{i 0} t+\delta n_{i} t+\sum_{\Phi^{*}} B_{i, \Phi^{*}} \sin \Phi^{*}+\sum_{\Phi} B_{i, \Phi} \sin \Phi \\
k_{i}= & \sum_{k=1}^{8} \lambda_{i k} M_{k} \cos \psi_{k}+\sum_{k=1}^{8} M_{i, \psi_{k}} \cos \psi_{k} \\
& +\sum_{\Phi^{*}} \epsilon_{\Phi^{*}} M_{i, \Phi^{*}} \cos \Phi^{*}+\sum_{\Phi} \epsilon_{\Phi} M_{i, \Phi} \cos \Phi
\end{aligned}
$$




$$
\begin{aligned}
h_{i}= & \sum_{k=1}^{8} \lambda_{i k} M_{k} \sin \psi_{k}+\sum_{k=1}^{8} M_{i, \psi_{k}} \sin \psi_{k} \\
& +\sum_{\Phi^{*}} M_{i, \Phi^{*}} \sin \Phi^{*}+\sum_{\Phi} M_{i, \Phi} \sin \Phi \\
q_{i}= & \sum_{k=1}^{8} \mu_{i k} N_{k} \cos \theta_{k}+\sum_{k=1}^{8} N_{i, \theta_{k}} \cos \theta_{k} \\
& +\sum_{\Phi^{*}} \epsilon_{\Phi^{*}} N_{i, \Phi^{*}} \cos \Phi^{*}+\sum_{\Phi} \epsilon_{\Phi} N_{i, \Phi} \cos \Phi \\
p_{i}= & \sum_{k=1}^{8} \mu_{i k} N_{k} \sin \theta_{k}+\sum_{k=1}^{8} N_{i, \theta_{k}} \sin \theta_{k} \\
& +\sum_{\Phi^{*}} N_{i, \Phi^{*}} \sin \Phi^{*}+\sum_{\Phi} N_{i, \Phi} \sin \Phi
\end{aligned}
$$

with :

$$
\Phi=\sum_{j=1}^{8} r_{j} \bar{\lambda}_{j}+\sum_{j=1}^{8} l_{j} \psi_{j}+\sum_{j=1}^{8} m_{j} \theta_{j}
$$

and

$$
\epsilon_{\Phi}=\operatorname{sign}\left(\sum_{j=1}^{8} r_{j}+\sum_{j=1}^{8} l_{j}+\sum_{j=1}^{8} m_{j}\right)
$$

$\overline{\lambda_{j}}, \psi_{j}, \theta_{j}$ represent, respectively, the argument of the longitude, the argument of the Lagrange solution in eccentricity, the argument of the Lagrange solution in inclination, connected to the planet $j$. The matrices $\left(\lambda_{i k}\right)$ and $\left(\mu_{i k}\right)$ are the matrices of the eigenvectors of the Lagrange solution. In the expressions (1), $\Phi^{*}$ arguments correspond to the case in which all the $r_{j}$ of the formula (2) are zeros, that is to say to the long period terms.

\subsection{LONG PERIOD VARIATIONS OF THE SEMI MAJOR AXIS AND OF THE LONGITUDE}

General theories are usually limited to the second order with respect to the disturbing masses and the quantities $A_{i, \Phi^{*}}$ of the formula (1) are zeros. In the study of the motion of the four outer planets Jupiter, Saturn, Uranus and Neptune, (Bretagnon and Simon, 1990), (Bretagnon and Francou, 1992) have determined, by an iterative method, perturbations of orders greater than 2 and thus obtained long period terms in the semi major axes. We find, for instance in the semi major axis of Saturn : 


$$
\begin{array}{rlr}
a_{\text {Saturn }}= & 9.554858819 \\
& +0.000188232 \cos \left(\psi_{5}-\psi_{6}\right) & \\
& +0.000022210 \cos \left(2 \psi_{5}-2 \psi_{6}\right) & 54017 \\
& +0.000015287 \cos \left(\psi_{6}-\psi_{7}\right) & 27009 \\
& +0.000004720 \cos \left(2 \psi_{6}-2 \theta_{6}\right) & 51540 \\
& +0.000003587 \cos \left(\psi_{5}-\psi_{7}\right) & 11873 \\
& +0.000003309 \cos \left(\psi_{5}-2 \psi_{6}+\psi_{7}\right) & 1124076 \\
& -0.000002358 \cos \left(2 \psi_{5}-\psi_{6}-\psi_{7}\right) & 56745 \\
& +\cdots
\end{array}
$$

In the formula (3), we have given the period of each term in years. By the third Kepler law, these terms produce long period terms with very large amplitude in the longitude. The formula (4) gives the most important long period perturbations of the longitude of Saturn as well as the most important short period terms.

$$
\begin{aligned}
\lambda_{\text {Saturn }}= & 0.927745+213.298190 t \\
& +0.055714 \sin \left(\psi_{5}-\psi_{6}\right) \\
& -0.012999 \sin \left(\psi_{5}-\psi_{7}\right) \\
& -0.011780 \sin \left(\psi_{5}-\psi_{6}-\theta_{6}+\theta_{7}\right) \\
& -0.004036 \sin \left(\psi_{6}-\psi_{7}\right) \\
& +0.003238 \sin \left(2 \psi_{5}-2 \psi_{6}\right) \\
& -0.000744 \sin \left(2 \psi_{5}-\psi_{6}-\psi_{7}\right) \\
& -0.000477 \sin \left(2 \psi_{5}-2 \psi_{6}-\theta_{6}+\theta_{7}\right) \\
& +0.000462 \sin \left(\psi_{5}-2 \psi_{6}+\psi_{7}\right) \\
& -0.000399 \sin \left(\theta_{6}-\theta_{7}\right) \\
& +\cdots \\
& +0.014273 \sin \left(2 \lambda_{5}-5 \lambda_{6}+3 \psi_{6}\right) \\
& +0.003917 \sin \left(2 \lambda_{5}-5 \lambda_{6}-\psi_{5}+4 \psi_{6}\right) \\
& +0.002594 \sin \left(\lambda_{5}-\lambda_{6}\right) \\
& +0.002199 \sin \left(2 \lambda_{5}-5 \lambda_{6}+\psi_{5}+2 \psi_{6}\right) \\
& +0.001172 \sin \left(2 \lambda_{5}-5 \lambda_{6}+\psi_{5}+3 \psi_{6}-\psi_{7}\right) \\
& -0.001108 \sin \left(2 \lambda_{5}-5 \lambda_{6}-\psi_{5}+3 \psi_{6}+\psi_{7}\right) \\
& -0.001070 \sin \left(\lambda_{5}-2 \lambda_{6}+\psi_{5}\right) \\
& +0.000934 \sin \left(\lambda_{5}-2 \lambda_{6}+\psi_{6}\right) \\
& +\cdots
\end{aligned}
$$

The amplitudes are in radians, time in thousands Julian years from J2000.

These long period perturbations of the longitudes with very large amplitudes are therefore essentially of the third order with respect to the masses. We give the more important ones, expressed in arcseconds, in table 1 for the longitudes of Jupiter and of Saturn, in table 2 for the ones of Uranus and of Neptune. 
TABLE 1. Amplitude of the long period terms in the longitudes of Jupiter and Saturn. The unit is the arcsecond.

\begin{tabular}{lrrr}
\hline Argument & Period & $B_{J, \Phi^{*}}$ & $B_{S, \Phi^{*}}$ \\
\hline$\psi_{5}-\psi_{6}$ & 54017 & -4653 & 11492 \\
$\psi_{5}-\psi_{7}$ & 1124076 & 1606 & -2681 \\
$\psi_{5}-\psi_{6}-\theta_{6}+\theta_{7}$ & 1996218 & 986 & -2430 \\
$\psi_{6}-\psi_{7}$ & 51540 & 345 & -832 \\
$2 \psi_{5}-2 \psi_{6}$ & 27009 & -272 & 668 \\
$2 \psi_{5}-\psi_{6}-\psi_{7}$ & 56744 & 62 & -154 \\
$2 \psi_{5}-2 \psi_{6}-\theta_{6}+\theta_{7}$ & 52594 & 40 & -98 \\
$\psi_{5}-2 \psi_{6}+\psi_{7}$ & 26375 & -39 & 95 \\
\hline
\end{tabular}

TABLE 2. Amplitude of the long period terms in the longitudes of Uranus and Neptune. The unit is the arcsecond.

\begin{tabular}{lrrr}
\hline Argument & Period & $B_{U, \Phi^{*}}$ & $B_{N, \Phi^{*}}$ \\
\hline$\psi_{5}-\psi_{7}$ & 1124076 & -20583 & 9996 \\
$\psi_{7}-\psi_{8}$ & 535721 & -431 & 263 \\
$\psi_{5}-\psi_{6}$ & 54017 & -248 & 13 \\
$\psi_{5}-\psi_{8}$ & 362810 & 220 & -250 \\
$\theta_{7}-\theta_{8}$ & 562640 & 159 & -43 \\
$\psi_{6}-\psi_{7}$ & 51540 & -97 & 22 \\
$2 \psi_{5}-2 \psi_{7}$ & 562038 & -13 & -34 \\
\hline
\end{tabular}

\subsection{LONG PERIOD VARIATIONS OF THE PLANETARY ORBITS}

The general theories are developed at the Bureau des Longitudes for all the planets since 1970 . They take into account the mutual perturbations of all the planets one another, the relativistic effects, the lunar perturbations. The long period terms of the variables $k, h, q, p$ give the variations of the planetary orbits over several millions years. These solutions bear a fundamental part in paleoclimatology because they allow to date with a great accuracy the glaciations of the Earth of the quaternary period (Berger, 1973), the climatic variations of Mars (Ward, 1979), (Borderies, 1980).

\section{Secular variation theories}

The general theories represent the motion of the planets over very large time spans but with a weak precision. The secular variation theories attempt to 
reach a great precision over time spans restricted to a few thousands of years. Therefore it is useless to keep the long period terms in a periodic form. By construction, we directly determine the time polynomial corresponding to the long period part of the formulas (1) and the Poisson series corresponding to the short period terms. Thus, to the long period terms of the formula (3) corresponds the time polynomial of the semi major axis of Saturn :

$$
\begin{aligned}
a_{\text {Saturn }}= & 9.5549091915-21.3896 \times 10^{-6} t+444 \times 10^{-10} t^{2} \\
& +670 \times 10^{-10} t^{3}+110 \times 10^{-10} t^{4}
\end{aligned}
$$

where $t$ is reckoned in thousands Julian years from J2000.

The first determinations of these secular terms of the semi major axes were obtained by (Simon and Chapront, 1974). Duriez (1978) has given a proof of the Poisson theorem and established that these terms are of order equal or greater than 3 with respect to the planetary masses.

The expansion of the long period terms with respect to time reduces considerably the size of the series and allows to reach a great precision. Thus, the longitude of Saturn of the formula (4) becomes :

$$
\begin{aligned}
\lambda_{\text {Saturn }}= & 0.874016284+213.299104960 t+0.000366597 t^{2} \\
& -806 \times 10^{-9} t^{3}-557 \times 10^{-9} t^{4} \\
& +0.013944575 \sin \left(2 \lambda_{5}-5 \lambda_{6}\right)+0.002196781 \cos \left(2 \lambda_{5}-5 \lambda_{6}\right) \\
& -0.001590423 t \sin \left(2 \lambda_{5}-5 \lambda_{6}\right)+0.005404368 t \cos \left(2 \lambda_{5}-5 \lambda_{6}\right) \\
& -0.001061109 t^{2} \sin \left(2 \lambda_{5}-5 \lambda_{6}\right)-0.000474470 t^{2} \cos \left(2 \lambda_{5}-5 \lambda_{6}\right) \\
& +\cdots
\end{aligned}
$$

In the formula (6), the coefficients are in radians.

The polynomial part of a variable is the mean element of this variable. It represents the development with respect to time of the long period part of the general theories. The mean element contains the most important variations but it does not represent a good approximation of the solution, particularly for the outer planets which include very large short period perturbations as we see in the expression (6).

The present analytical theories VSOP82 (Bretagnon, 1982), TOP82 (Simon, 1983), VSOP87 (Bretagnon and Francou, 1988) have, over one century around J2000, an accuracy of about $10^{-7}$ for Jupiter and Saturn and of about $10^{-8}$ for the other planets. They include the mutual perturbations of all the planets one another up to an order with respect to the masses equal or greater than 3 , the relativistic contributions of the Schwarzschild problem, the perturbations by the Moon and some asteroids. They are computed using the planetary mass values of IAU (Grenoble, 1976); the 
integration constants are determined by comparison to DE200 numerical integration of JPL (Standish, 1982).

The precisions of these solutions seem good enough for the two following applications : the determination of the difference between the barycentric time $T C B$ and the geocentric time $T C G$ and the computation of the terms of precession and of nutation for the rigid Earth.

\section{Relation between $T C B$ and $T C G$}

The analytical solutions of the motion of the planets and of the Moon ELP2000/82 (Chapront-Touzé and Chapront, 1983) were used in the computation of the relation between the Barycentric Coordinate Time (TCB) and the Geocentric Coordinate Time (TCG) par (Hiramaya et al 1987), (Fairhead and Bretagnon, 1990).

Restricted to the terms proportional to $c^{-2}$, the relation between $T C B$ and $T C G$ is written :

$$
\begin{aligned}
\mathrm{TCB}= & \mathrm{TCG}+c^{-2} \int_{T C B_{0}}^{T C B}\left(U_{E}+\frac{1}{2} v_{E}^{2}\right) \mathrm{d}\left(T C B^{\prime}\right) \\
= & \mathrm{TCG}+L_{C} T C B \\
& +1656.674564 \mu \mathrm{s} \sin (6283.075850 T C B+6.240054)+\cdots
\end{aligned}
$$

with $L_{C}=1.4808268475 \times 10^{-8}$ and $T C B$ in thousands Julian years.

$U_{E}$ represents the external mass force function evaluated at the geocentre and has been computed taking into account the Sun, the Moon and the planets from Mercury to Neptune.

By comparison to numerical integrations, T. Fukushima and A. Irwin have shown that this solution has an accuracy of $1.8 \mathrm{~ns}$ over (1980-2000). At the beginning, the solution of (Fairhead and Bretagnon, 1990) retained only the periodic terms greater than $0.1 \mathrm{~ns}$. To obtain the precision of $1.8 \mathrm{~ns}$, we have taken into account the 971 periodic terms greater than $0.01 \mathrm{~ns}$.

To improve the relation between $T C B$ and $T C G$, we have :

- to compute $U_{E}$ and $v_{E}$ with planetary motion solutions using recent values of the planetary masses, for instance the ones of the IERS Standards 1992 (McCarthy, 1992);

- to take into account Pluto : $\Delta L_{C}^{\text {Pluto }} \sim 2 \times 10^{-18}$;

- to take into account asteroids (Fukushima, 1995) : $\Delta L_{C}^{A} \sim 4.5 \times 10^{-18}$;

- to determine the terms proportional to $c^{-4}$. For these terms, (Moisson, 1995) finds :

$$
\begin{aligned}
\Delta(T C B-T C G)= & c^{-4} \int_{T C B_{0}}^{T C B}\left(\frac{1}{8} v_{E}^{4}+\frac{3}{2} U_{E} v_{E}^{2}-\frac{1}{2} U_{E}^{2}\right) \mathrm{d}\left(T C B^{\prime}\right) \\
= & 1.0965 \times 10^{-16} T C B-0.10 \times 10^{-20} T C B^{2} \\
& -7^{s} .3 \times 10^{-12} \sin \left(\lambda_{3}\right)-31^{s} .9 \times 10^{-12} \cos \left(\lambda_{3}\right)+\cdots
\end{aligned}
$$


with $T C B$ in thousands Julian years.

\section{Precession and nutation for the rigid Earth}

\subsection{EQUATIONS OF THE MOTION}

We have established the motion equations for the rigid Earth with the Euler angles $\psi, \omega, \varphi$ reckoned in the positive direction and $\omega$ being the rotation angle from ecliptic J2000 to the equator of date. We have therefore :

$$
\begin{aligned}
\psi & =-\psi_{A} \\
\omega & =-\omega_{A}
\end{aligned}
$$

where $\psi_{A}$ and $\omega_{A}$ represent the luni-solar precession and the obliquity with the notations of (Lieske et al 1977). The sidereal time $\varphi$ is given by :

with :

$$
\varphi=\varphi_{0}+\varphi_{1} t+\Delta \varphi
$$

$$
\begin{aligned}
& \varphi_{0}=4.90356257935 \\
& \varphi_{1}=2301216.7531542 \mathrm{rd} / \text { thousand Julian years }
\end{aligned}
$$

We also defined :

$$
\tilde{\varphi}=\varphi+\alpha
$$

with :

$$
\alpha=-14^{\circ} .95
$$

longitude of major axis of equatorial ellipse (Bursa, 1992).

Then, the equations are written :

$$
\begin{aligned}
\ddot{\omega}+\frac{C}{A} \sin \omega_{0} \varphi_{1} \dot{\psi} & =\frac{L}{A}+F_{2}+\frac{B-A}{A} F_{1} \\
\sin \omega_{0} \ddot{\psi}-\frac{C}{A} \varphi_{1} \dot{\omega} & =\frac{M}{A}+G_{2}+\frac{B-A}{A} G_{1} \\
\ddot{\varphi} & =\frac{N}{C}+H_{2}+\frac{B-A}{C} H_{1}
\end{aligned}
$$

with :

$$
\begin{aligned}
F_{2} & =-\frac{C}{A} \dot{\psi} \varphi_{1}\left(\sin \omega-\sin \omega_{0}\right)-\frac{C}{A} \dot{\psi} \Delta \dot{\varphi} \sin \omega-\frac{C-A}{A} \dot{\psi}^{2} \sin \omega \cos \omega \\
G_{2} & =-\ddot{\psi}\left(\sin \omega-\sin \omega_{0}\right)+\frac{C}{A} \dot{\omega} \Delta \dot{\varphi}-\frac{A+B-C}{A} \dot{\psi} \dot{\omega} \cos \omega \\
H_{2} & =-\ddot{\psi} \cos \omega+\dot{\psi} \dot{\omega} \sin \omega
\end{aligned}
$$

and : 


$$
\begin{array}{ll}
F_{1}= & \ddot{\psi} \sin \tilde{\varphi} \cos \tilde{\varphi} \sin \omega+\dot{\psi} \dot{\varphi}\left(\cos ^{2} \tilde{\varphi}-\sin ^{2} \tilde{\varphi}\right) \sin \omega-2 \dot{\omega} \dot{\varphi} \sin \tilde{\varphi} \cos \tilde{\varphi} \\
& -\ddot{\omega} \sin ^{2} \tilde{\varphi}+\dot{\psi}^{2} \cos ^{2} \tilde{\varphi} \sin \omega \cos \omega \\
G_{1}= & 2 \dot{\psi} \dot{\varphi} \sin \tilde{\varphi} \cos \tilde{\varphi} \sin \omega+\ddot{\omega} \sin \tilde{\varphi} \cos \tilde{\varphi}+\dot{\omega} \dot{\varphi}\left(\cos ^{2} \tilde{\varphi}-\sin ^{2} \tilde{\varphi}\right) \\
& +\dot{\psi}^{2} \sin \tilde{\varphi} \cos \tilde{\varphi} \sin \omega \cos \omega-\ddot{\psi} \cos ^{2} \tilde{\varphi} \sin \omega \\
H_{1}= & \dot{\omega}^{2} \sin \tilde{\varphi} \cos \tilde{\varphi}-\dot{\psi} \dot{\omega}\left(\cos ^{2} \tilde{\varphi}-\sin ^{2} \tilde{\varphi}\right) \sin \omega-\dot{\psi}^{2} \sin \tilde{\varphi} \cos \tilde{\varphi} \sin ^{2} \omega
\end{array}
$$

Let $O x y z$ be the reference frame ecliptic J2000 and $O X Y Z$ the nonrotating equator of date. From $O x y z$, we define $O X Y Z$ with 2 rotations:

- a rotation with $\psi$ angle around $z$ axis;

- a rotation with $\omega$ angle around $X$ axis.

The quantities $L, M, N$ of the formula (7) represent thus the components, in $O X Y Z$, of the torque of the external forces with respect to the geocenter $O$. $A, B, C$ are the moments of inertia.

\subsection{USED MODEL}

In the computation of the quantities $L, M, N$, we take into account, for the influence by the Moon, the terms of the terrestrial potential depending on $C_{n, 0}$ for $n$ from 2 to 5 , on $C_{2,2}, S_{2,2}$ and $C_{3, k}, S_{3, k}$ for $k$ from 1 to 3 ; for the influence by the Sun : $C_{2,0}, C_{3,0}, C_{2,2}, S_{2,2}, C_{3,1}, S_{3,1}$; for the influence by the planets from Mercury to Neptune : $C_{2,0}$. The lunar theory used is ELP2000/82 (Chapront-Touzé and Chapront, 1983); the one of the planets and the Sun is VSOP87A (Bretagnon and Francou, 1988). In this study, we take the following choices :

a) We study the variations of the rigid Earth equator with respect to the ecliptic and the equinox J2000. So, we have to take into account the perturbations of the equator by the Moon, the Sun and the planets, the motion of which is expressed in rectangular coordinates with respect to the ecliptic and the equator $\mathrm{J} 2000$.

b) The solution is expanded in Fourier and Poisson series, the angles of which are linear combinations of the planet longitudes $\lambda_{i}$ reckoned from the equinox J2000 and of the Delaunay angles which do not depend on the origin. In consequence, the 18.6 year period perturbation is represented by the angle $\lambda_{3}+D-F$ which differs from the longitude $\Omega$ of the node of the Moon referred to the equinox of date :

$$
\lambda_{3}+D-F=\Omega+180^{\circ}-p \times t
$$

where $p$ is the constant of the precession in longitude and $t$ the time reckoned from J2000.

c) In the Fourier and Poisson series, we keep only the linear part of the mean longitudes of the planets and of the Delaunay angles $D, F, l_{M}$; the poly- 
nomial parts, the degree of which is equal or greater than 2 are expanded in Poisson series.

d) The perturbations due to the Moon are computed as a whole. We do use a representation of the lunar motion in rectangular coordinates, containing the perturbations of the main problem, the direct and indirect planetary perturbations, the perturbations due to the terrestrial potential, the tidal effects. In the same way, we use a solution of the Sun in rectangular coordinates reckoned with respect to the Earth but not to the Earth-Moon barycenter.

e) The computation was performed with the value of the precession constant given by (Williams et al 1991) and used by (Simon et al 1994) :

$$
p=50288^{\prime \prime} .200 / \text { thousand Julian years. }
$$

This value corresponds to :

$$
\left(\frac{\mathrm{d} \psi_{A}}{\mathrm{~d} t}\right)_{t=0}=50385^{\prime \prime} .0672 .
$$

The value of the geodesic precession $p_{g}$ determined by (Brumberg et al 1991) is :

$$
p_{g}=19^{\prime \prime} .1988 \text {. }
$$

We have therefore solved equations (7) fixing the value of the moment of inertia $C$ :

$$
C=1.805465872 \times 10^{-15}\left(\mathrm{~m}_{\mathrm{S}} \mathrm{au}^{2}\right)
$$

in order to obtain :

$$
-\left(\frac{\mathrm{d} \psi}{\mathrm{d} t}\right)_{t=0}=50385^{\prime \prime} .0672+19^{\prime \prime} .1988=50404^{\prime \prime} .2660 .
$$

The value of the obliquity is :

$$
\varepsilon_{0}=23^{\circ} 26^{\prime} 21^{\prime \prime} .412 \text {. }
$$

In the relation (10), $m_{S}$ is the mass of the Sun :

$$
m_{S}=332946.045 m_{E}
$$

At this value of $C$ corresponds the dynamical ellipticity $H_{d}$ :

$$
H_{d}\left(50288^{\prime \prime} .2\right)=0.00327380045
$$

For $p=50287^{\prime \prime} .7$, one obtains :

$$
H_{d}\left(50287^{\prime \prime} .7\right)=0.00327376798
$$


TABLE 3. Most important perturbations of the nutation. Amplitudes are in $10^{-6}$ ", periods in days.

\begin{tabular}{|c|c|c|c|c|}
\hline Origin & Argument & Amplitude in $\psi$ & Amplitude in $\omega$ & Period \\
\hline Moon $C_{2,0}$ & $\lambda_{3}+D-F$ & 17292345.65 & 9227970.05 & 6793.48 \\
\hline$C_{3,0}$ & $\lambda_{3}+D-l$ & 104.05 & 88.95 & 3232.61 \\
\hline$C_{4,0}$ & $\lambda_{3}+D-F$ & 0.73 & 6.84 & 6793.48 \\
\hline$C_{5,0}$ & $\lambda_{3}+D-l$ & 0.01 & 0.00 & 3232.61 \\
\hline$C_{2,2}-S_{2,2}$ & $2 \lambda_{3}+2 D-2 \varphi$ & 29.44 & 11.71 & 0.52 \\
\hline$C_{3,1}-S_{3,1}$ & $\lambda_{3}+D+\varphi$ & 38.44 & 15.25 & 0.96 \\
\hline$C_{3,2}-S_{3,2}$ & $\lambda_{3}+D-2 \varphi$ & 0.39 & 0.14 & 0.51 \\
\hline$C_{3,3}-S_{3,3}$ & $\lambda_{3}+D-3 \varphi$ & 0.41 & 0.20 & 0.34 \\
\hline Sun $C_{2,0}$ & $2 \lambda_{3}$ & 1276723.69 & 552395.17 & 182.63 \\
\hline$C_{3,0}$ & $\lambda_{3}$ & 0.26 & 0.22 & 365.26 \\
\hline$C_{2,2}-S_{2,2}$ & $2 \lambda_{3}-2 \varphi$ & 12.32 & 4.90 & 0.50 \\
\hline$C_{3,1}-S_{3,1}$ & $\lambda_{3}+\varphi$ & 2.79 & 1.11 & 1.00 \\
\hline Mercury $C_{2,0}$ & $\lambda_{1}-4 \lambda_{3}$ & 1.03 & 0.43 & 2432.11 \\
\hline Venus $C_{2,0}$ & $3 \lambda_{2}-5 \lambda_{3}$ & 216.71 & 90.76 & 2959.21 \\
\hline $\operatorname{Mars} C_{2,0}$ & $\lambda_{3}-2 \lambda_{4}$ & 11.55 & 0.95 & 5764.01 \\
\hline Jupiter $C_{2,0}$ & $2 \lambda_{5}$ & 104.41 & 45.69 & 2166.29 \\
\hline Saturn $C_{2,0}$ & $2 \lambda_{6}$ & 12.15 & 5.16 & 5379.61 \\
\hline Uranus $C_{2,0}$ & $2 \lambda_{7}$ & 0.65 & 0.29 & 15344.24 \\
\hline Neptune $C_{2,0}$ & $2 \lambda_{8}$ & 0.40 & 0.16 & 30091.15 \\
\hline Complements & $\lambda_{3}+D-F$ & 15361.43 & 0.04 & 6793.48 \\
\hline
\end{tabular}

\subsection{RESULTS}

We give in table 3 the most important perturbation of each component. For the planets, it is the direct influence which is concerned. Complements correspond to the quantities $F_{1}, G_{1}, H_{1}, F_{2}, G_{2}, H_{2}$ of the equations (7). Table 4 gives the different components to the secular terms of $\psi_{A}$ and of $\omega_{A}$. In table (5) we compare the polynomial parts of $\psi_{A}$ and $\omega_{A}$ to the results of (Simon et al 1994) and of (Williams, 1994). The difference with Williams et al in $\psi_{A}(t)$ results from a different choice of precession constant and an insufficient model in Simon et al explains the difference in $\omega_{A}(t)$. Besides, Williams takes into account the secular variation of $J_{2}$ that explains the discrepancy in $\psi_{A}\left(t^{2}\right)$. With the value of $\dot{J}_{2}$ (Bursa, 1992) :

$$
\dot{J}_{2}=(-2.8 \pm 0.3) \times 10^{-9} / \text { century }
$$

we compute the following perturbation :

$$
\begin{aligned}
\Delta \psi_{A}\left(\dot{J}_{2}\right)= & -0^{\prime \prime} .651804 t^{2}+0^{\prime \prime} .001849 t^{3}+0^{\prime \prime} .000022 t^{4}+\cdots \\
& -0^{\prime \prime} .000447 t \sin \left(\lambda_{3}+D-F\right)+\cdots
\end{aligned}
$$


TABLE 4. Secular term of $\psi_{A}$ and of $\omega_{A}$ in arcseconds per thousand years.

\begin{tabular}{rrr}
\hline Origin & $\psi_{A}$ & $\omega_{A}$ \\
\hline Moon $C_{2,0}$ & 34455.298798 & -0.254417 \\
$C_{3,0}$ & -0.000057 & -0.000011 \\
$C_{4,0}$ & 0.025192 & \\
Sun $C_{2,0}$ & 15948.860274 & 0.002923 \\
$C_{3,0}$ & -0.000026 & -0.000005 \\
Mercury $C_{2,0}$ & 0.003698 & -0.000088 \\
Venus $C_{2,0}$ & 0.181582 & -0.016814 \\
Mars $C_{2,0}$ & 0.005999 & 0.000357 \\
Jupiter $C_{2,0}$ & 0.117060 & 0.002804 \\
Saturn $C_{2,0}$ & 0.005208 & 0.000220 \\
Uranus $C_{2,0}$ & 0.000100 & 0.000001 \\
Neptune $C_{2,0}$ & 0.000029 & 0.000001 \\
Complements & -0.231857 & \\
$-p_{g}$ & -19.198800 & \\
\hline & 50385.067200 & -0.265029 \\
\hline
\end{tabular}

$$
\begin{aligned}
\Delta \omega_{A}\left(\dot{J}_{2}\right)= & 0^{\prime \prime} .000003 t^{2}-0^{\prime \prime} .000088 t^{3}+0^{\prime \prime} .000150 t^{4}+\cdots \\
& +0^{\prime \prime} .000239 t \cos \left(\lambda_{3}+D-F\right)+\cdots
\end{aligned}
$$

TABLE 5. Secular variations of $\psi_{A}$ and of $\omega_{A}$.

\begin{tabular}{lrcll}
\hline & $t$ & $t^{2}$ & \multicolumn{1}{c}{$t^{3}$} & $t^{4}$ \\
\hline$\psi_{A}$ & 50385.067200 & -107.246837 & -1.144309 & 1.329708 \\
Simon et al & 50385.067200 & -107.2374 & -1.1424 & 1.3279 \\
Williams & 50384.565010 & -107.8977 & -1.141 & 1.33 \\
$\omega_{A}$ & -0.265029 & 5.129643 & -7.732154 & -0.004852 \\
Simon et al & & 5.1294 & -7.7276 & -0.0048 \\
Williams & -0.24400 & 5.1268 & -7.727 & \\
\hline
\end{tabular}

From $\psi_{A}$ and $\omega_{A}$, we have computed the variables $p_{A}$ and $\varepsilon_{A}$ and compared to the KS solution of (Kinoshita and Souchay, 1990). Kinoshita and Souchay use the following value of the precession constant :

$$
p=50290 " .966 .
$$

So, we have multiplied their solution by :

$$
\text { coef }=\frac{50404.266}{50407.032}
$$


TABLE 6. Difference $p_{A}-$ coef KS. Unit is $10^{-6 \prime \prime}$.

\begin{tabular}{lrrc}
\hline Argument & sin & cos & Period \\
\hline $2 \lambda_{5}-5 \lambda_{6}$ & -499 & -660 & $883 \mathrm{y}$ \\
$3 \lambda_{3}$ & 216 & 888 & $121.75 \mathrm{~d}$ \\
$\lambda_{3}+D-F$ & -25 & -384 & $6793.48 \mathrm{~d}$ \\
$2 \lambda_{3}+2 D-F-l_{M}$ & 20 & 281 & $6167.21 \mathrm{~d}$ \\
$4 \lambda_{3}-8 \lambda_{4}+3 \lambda_{5}$ & -52 & 166 & $1783 \mathrm{y}$ \\
$\lambda_{3}+D-l_{M}$ & -106 & 96 & $3232.61 \mathrm{~d}$ \\
$8 \lambda_{2}-13 \lambda_{3}$ & 79 & 31 & $239 \mathrm{y}$ \\
$2 \lambda_{3}+2 D$ & 81 & -3 & $13.66 \mathrm{~d}$ \\
$F-l_{M}$ & -33 & 33 & $2190.35 \mathrm{~d}$ \\
\hline
\end{tabular}

in order to make the solutions comparables. We give in table 6 the most important discrepancies $p_{A}-$ coef KS.

\section{References}

Berger, A. (1973) Théorie astronomique des paléoclimats, Thèse, Louvain.

Borderies, N. (1980) La rotation de Mars. Thèse, Toulouse.

Bretagnon, P. (1982) Astron. Astrophys., 114, p. 278-288.

Bretagnon, P., Francou, G. (1988) Astron. Astrophys., 202, p. 309-315.

Bretagnon, P., Simon, J.L. (1990) Astron. Astrophys., 239, p. 387-398.

Bretagnon, P., Francou, G. (1992) IAU Symposium no. 152, p. 37-42.

Brumberg, V.A., Bretagnon, P., Francou, G. (1991) in : Capitaine N. (ed.) Systèmes de références spatio-temporels. Journées 1991, Obs. de Paris, p. 141.

Bursa, M. (1992) Bull. Geod., 66-2, p. 193.

Chapront-Touzé, M., Chapront, J. (1983) Astron. Astrophys., 124, p. 50.

Duriez, L. (1978) Astron. Astrophys., 68, p. 199.

Fairhead, L., Bretagnon, P. (1990) Astron. Astrophys., 229, p. 240.

Fukushima, T. (1995) Astron. Astrophys., 294, p. 895.

Hirayama, Th., Fujimoto, M.-K., Kinoshita, H., Fukushima, T. (1987) IAG Symposia at IUGG, Tome I, p. 91

Kinoshita, H., Souchay, J. (1990) Celest. Mech., 48, p. 187.

Lieske, J.H., Lederle, T., Fricke, W., Morando, B. (1977) Astron. Astrophys., 58, p. 1-16. McCarthy, D.D. (1992), IERS Standards (1992) IERS Technical Note 13, Observatoire de Paris.

Moisson, X. (1995), Rapport de stage de DEA Observatoire de Paris.

Simon, J.-L., Chapront, J. (1974) Astron. Astrophys., 32, p. 51.

Simon, J.L. (1983) Astron. Astrophys., 120, p. 197-202.

Simon, J.L., Bretagnon, P., Chapront, J., Chapront-Touzé, M., Francou, G., Laskar, J. (1994) Astron. Astrophys., 282, p. 663-683.

Standish, E.M. (1982) Astron. Astrophys., 114, p. 297-302.

Ward, W.R. (1979) Present Obliquity Oscillations of Mars : Fourth-Order Accuracy on Orbital e and I. J. Geophys. Res., 84 p. 237.

Williams, J. G., Newhall X X, Dickey, J. O. (1991) Astron. Astrophys., 241, L9.

Williams, J. G. (1994) Astron. J., 108 (2), p. 711. 